\title{
Downregulation of miR-204 expression defines a highly aggressive subset of Group 3/Group 4 medulloblastomas
}

Harish Shrikrishna Bharambe ${ }^{1,10+}$, Raikamal Paul ${ }^{1,10+}$, Pooja Panwalkar ${ }^{1,10+}$, Rakesh Jalali ${ }^{4}$, Epari Sridhar ${ }^{5}$, Tejpal Gupta ${ }^{2}$, Aliasgar Moiyadi ${ }^{6}$, Prakash Shetty ${ }^{6}$, Sadaf Kazi ${ }^{1}$, Akash Deogharkar ${ }^{1,10}$, Shalaka Masurkar ${ }^{1,10,}$ Kedar Yogi ${ }^{1,10}$, Ratika Kunder ${ }^{1,10}$, Nikhil Gadewal ${ }^{3}$, Atul Goel ${ }^{8}$, Naina Goel ${ }^{9}$, Girish Chinnaswamy ${ }^{7}$, Vijay Ramaswamy ${ }^{11^{*}}$ and Neelam Vishwanath Shirsat ${ }^{1,10^{*}}$

\begin{abstract}
Genome-wide expression profiling studies have identified four core molecular subgroups of medulloblastoma: WNT, SHH, Group 3 and Group 4. Molecular markers are necessary for accurate risk stratification in the nonWNT subgroups due to the underlying heterogeneity in genetic alterations and overall survival. MiR-204 expression was evaluated in molecularly classified 260 medulloblastomas from an Indian cohort and in 763 medulloblastomas from the MAGIC cohort, SickKids, Canada. Low expression of miR-204 in the Group $3 /$ Group 4 tumors identify a highly aggressive subset of tumors having poor overall survival, in the two independent cohorts of medulloblastomas. Downregulation of miR-204 expression correlates with poor survival within the Group 4 as well indicating it as a valuable risk-stratification marker in the subgroup. Restoration of miR-204 expression in multiple medulloblastoma cell lines was found to inhibit their anchorage-independent growth, invasion potential and tumorigenicity. IGF2R was identified as a novel target of miR-204. MiR-204 expression resulted in downregulation of both M6PR and IGF2R that transport lysosomal proteases from the Golgi apparatus to the lysosomes. Consistent with this finding, miR-204 expression resulted in reduction in the levels of the lysosomal proteases in medulloblastoma cells. MiR-204 expression also resulted in inhibition of autophagy that is known to be dependent on the lysosomal degradation pathway and LC3B, a known miR-204 target. Treatment with HDAC inhibitors resulted in upregulation of miR204 expression in medulloblastoma cells, suggesting therapeutic role for these inhibitors in the treatment of medulloblastomas. In summary, miR-204 is not only a valuable risk stratification marker in the combined cohort of Group 3 / Group 4 medulloblastomas as well as in the Group 4 itself, that has paucity of good prognostication markers, but also has therapeutic potential as indicated by its tumor suppressive effect on medulloblastoma cells.
\end{abstract}

Keywords: Medulloblastoma, MiR-204, Risk stratification, Tumor-suppression, Autophagy

\footnotetext{
* Correspondence: vijay.ramaswamy@sickkids.ca; nshirsat@actrec.gov.in

${ }^{+}$Harish Shrikrishna Bharambe, Raikamal Paul and Pooja Panwalkar

contributed equally to this work.

${ }^{11}$ Division of Haematology/Oncology, Department of Paediatrics, Hospital for Sick Children and University of Toronto, 555 University Ave, Toronto, ON M5G 1X8, Canada

'Shirsat Laboratory, Advanced Centre for Treatment, Research \& Education in Cancer, Tata Memorial Centre, Kharghar, Navi Mumbai 410210, India Full list of author information is available at the end of the article
}

(c) The Author(s). 2019 Open Access This article is distributed under the terms of the Creative Commons Attribution 4.0 International License (http://creativecommons.org/licenses/by/4.0/), which permits unrestricted use, distribution, and reproduction in any medium, provided you give appropriate credit to the original author(s) and the source, provide a link to the Creative Commons license, and indicate if changes were made. The Creative Commons Public Domain Dedication waiver (http://creativecommons.org/publicdomain/zero/1.0/) applies to the data made available in this article, unless otherwise stated. 


\section{Introduction}

Brain tumors are the second most common cancers in children and the leading cause of cancer-related mortality in this age group [40]. Medulloblastoma, a highly malignant tumor of the posterior fossa region of the brain, is the single most common pediatric malignant brain tumor. Genome-wide expression profiling studies have identified four core molecular subgroups of medulloblastomas: WNT, SHH, Group 3 and Group 4 that are not only distinct in their underlying genetic alterations but also differ in clinical characteristics like age, gender related incidence, incidence of metastasis and overall survival rates [36]. WNT subgroup medulloblastomas that are characterized by the activation of the canonical WNT signaling pathway have excellent (>95\%) long term survival [44]. SHH subgroup medulloblastomas having activated Sonic Hedgehog signaling expression profile, have intermediate survival rates with those harboring mutation in the TP53 tumor suppressor gene or amplification of $M Y C N$ oncogene having poor survival [44]. The two non-WNT, non-SHH subgroups have some overlap in their expression profiles with a number of transcription factors involved in neural development being overexpressed in both the subgroups [37]. The two subgroups are distinguished based on the preferential expression of proliferation related genes, retina-specific genes in the Group 3 tumors and neuronal differentiation related genes in the Group 4 tumors [37]. Group 3 tumors have the worst survival rates among all the four subgroups while Group 4 tumors have intermediate survival rate. NRL and CRX, the two retina-specific transcription factors have been found to be master regulators of photoreceptor signaling program in the Group 3 medulloblastomas [9]. MYC amplifications are restricted to Group 3 [38]. Structural variants leading to aberrant induction of GFI1/GFI1B oncogenes and $M Y C N$ amplifications are found in both Group 3 and Group 4. Pathway analysis of recurrent genetic alterations have found overrepresentation of genes involved in the TGF $\beta$ and Notch signaling pathway in Group 3 and chromatin modifiers in Group 4 [34].

Surgery followed by radiation therapy and chemotherapy is the standard multimodal treatment for medulloblastoma [1]. Long term sequelae of the intense treatment include neurocognitive impairment, endocrine dysfunction, psychiatric, developmental deficits and in some cases secondary malignancies [17]. Accurate risk stratification of medulloblastomas is therefore necessary to spare the children having low risk of recurrence from excessive treatment to the developing brain. On the other hand, survival of high risk medulloblastoma cases can be improved by more aggressive treatment. Considerable heterogeneity exists in each of the three non-WNT subgroups for which molecular markers are necessary so that accurate risk stratification can be done for effective treatment with least side effects [44].

MicroRNAs are small non-coding molecules that have been shown to regulate a wide array of cell functions, ranging from cell proliferation, differentiation, cell death and stress resistance. Since the first report of miR-15/ miR-16 deletion in B cell chronic lymphocytic leukemia, large number of studies have reported microRNA dysregulation in cancer including medulloblastoma $[5,56]$. We have earlier reported differential expression profiles of microRNAs in the molecular subgroups of medulloblastomas [13]. Further, we have developed an assay based on the microRNA profile that has 97\% accuracy for molecular classification of medulloblastomas and is particularly useful for formalin-fixed, paraffin-embedded (FFPE) tumor tissues [19]. In the present study, miR-204 expression was analyzed in 260 medulloblastomas from an Indian cohort and in 763 medulloblastomas from the MAGIC (Medulloblastoma Advanced Genomics International Consortium) cohort [2]. A subset of Group 3 / Group 4 medulloblastomas having low expression of miR-204 was found to have significantly poor survival. The role of miR-204 expression in medulloblastoma biology was investigated by restoring miR-204 expression in established medulloblastoma cell lines and studying its effect on growth and malignant behavior of medulloblastoma cells.

\section{Materials and methods}

\section{Human tissue samples}

Medulloblastoma tumor tissues either as fresh frozen or FFPE tissues were obtained after acquiring informed consent from the patients. The study was approved by the Institutional Ethics Committee of the Tata Memorial Centre. The tumor tissues were snap-frozen in liquid nitrogen immediately after surgical resection and stored at $-80^{\circ} \mathrm{C}$. The histopathological diagnosis and grading of the tumor tissues was done as per the World Health Organization 2007 classification of tumors of the Central Nervous System [25] and only the tumors diagnosed as medulloblastomas were included in the study. Normal human brain tissues were obtained from the Human Brain Tissue Repository at the National Institute of Mental Health and Neurosciences, Bengaluru, India.

\section{Analysis of miR-204 expression}

Molecular classification of 260 medulloblastomas from the Indian cohort was carried out using real time RT-PCR (Reverse Transcription-Polymerase Chain Reaction) assay as described before [19]. MiR-204 expression was determined by the Taqman assay. RNU48 was used as a house-keeping small RNA control. Relative Quantity (RQ) was estimated as $\mathrm{RQ}=2^{-}{ }_{\text {test }}^{\left(\mathrm{Ct}_{\text {control }}\right)} \mathrm{X}$ 100. In the MAGIC validation cohort, miR-204 expression was 
analyzed across 763 primary medulloblastoma samples, profiled on the Affymetrix Gene 1.1 ST array as described previously, normalized using the RMA (Robust Multi-array Average) method and, subgrouped / subtyped using similarity network fusion (GSE85217) [2]. Differences across subgroups and subtypes were evaluated using ANOVA (Analysis of variance) in the $R$ statistical environment (v3.4.2). Survival was measured from the time of initial diagnosis to the date of death or last follow up. Survival distribution was estimated according to the Kaplan-Meier method using optimal cut-off selection and log-rank statistics using the survival package (v2.40-1) in the $\mathrm{R}$ statistical environment (v3.4.2). $P$ values $<0.01$ were considered to be statistically significant.

\section{Cell culture}

Human medulloblastoma cell line D283 was obtained from ATCC (American Type Culture Collection), Manassas, VA, USA. Authenticity of the cell lines was confirmed by the Short Tandem Repeat (STR) marker profiling before initiating the experiments. Medulloblastoma cell lines D425, D341 are kind gifts from Dr. Darell Bigner, Duke University Medical Centre, Durham, NC, USA. HD-MB03 cell line is a kind gift from Dr. Till Milde, German Cancer Research Centre, Germany. All the cell lines were checked for the presence of mycoplasma contamination by PCR based assay [53]. The cells were grown in Dulbecco's Modified Eagle Medium: Nutrient Mixture F-12 (DMEM/ F-12) supplemented with $10 \%$ Fetal Bovine Serum (FBS) in a humidified atmosphere of $5 \% \mathrm{CO}_{2}$.

\section{Restoration of miR-204 expression in medulloblastoma cells}

Genomic region encoding miR-204 was amplified from normal human lymphocyte DNA by PCR and cloned in pTRIPZ lentiviral vector downstream of doxycyline-inducible minimal Cytomegalo virus (CMV) promoter (Additional file 1: Table S1). The medulloblastoma cell lines were transduced with the pTRIPZ-miR-204 lentiviral particles and stable polyclonal populations were selected in the presence of puromycin. The cells transduced with lentiviral particles of empty pTRIPZ vector (Dharmacon, Lafayette, CO, USA) were used as vector control.

\section{Effect of miR-204 expression on proliferation and anchorage-independent growth}

Growth of miR-204 expressing cells and control cells was studied by the MTT reduction assay as described before [32, 59]. 2000 cells of the medulloblastoma cell lines were seeded per well of a 96-well micro-titer plate. Cell growth was followed over a period of 10-12 days with replenishment of medium every $3^{\text {rd }}$ day. For studying anchorage-independent growth by soft agar colony formation assay, 2000 cells were seeded in DMEM/F12 medium supplemented with $10 \%$ FBS containing 0.3\% agarose over a basal layer of $1 \%$ agarose in DMEM-F12/10\% FBS. The cells were incubated for about 1-2 weeks and the colonies formed were counted.

\section{Invasion assay}

75,000 cells of D283 / HD-MB03 cell line were seeded in $200 \mu \mathrm{l}$ of serum-free DMEM / F12 medium in the upper chamber of $8-\mu \mathrm{m}$ pore size transwell inserts (BD Biosciences, San Hose, CA, USA) coated with Matrigel ${ }^{\mathrm{m}}$, placed in a 24 well micro-titre plate. $750 \mu$ l of the medium supplemented with $10 \%$ FBS was added to the lower chamber. The cells were allowed to migrate for 56 $\mathrm{h}$ to $72 \mathrm{~h}$ depending upon the cell line and then labeled with Calcein-AM (Life technologies, Carlsbad, CA, USA), a fluorescent dye, $30 \mathrm{~min}$ prior to terminating the invasion. Non-invaded cells from the upper chamber were removed by wiping the upper portion of the insert with a cotton bud. The inserts were photographed using a Zeiss Axiovert $200 \mathrm{M}$ fluorescence microscope. Fluorescence intensity of the Calcein-AM labeled cells on the lower side of the insert was measured using a Mithras LB940 multimode reader (Berthhold Technologies, Bad Wildbad, Germany) using excitation wavelength of 485 $\mathrm{nm}$ and emission wavelength of $535 \mathrm{~nm}$.

\section{Tumorigenicity assay}

The experimental protocols were approved by the Institutional Animal ethics committee. Medulloblastoma cells were transduced with lentiviral particles of pCS-CG vector (a gift from Inder Verma, Addgene plasmid \#12154 [30]) expressing firefly luciferase cDNA FL2 (from pCAG-luciferase vector, a gift from Snorri Thorgeirsson, Addgene plasmid \#55764 [21]) under the CMV promoter. $2 \times 10^{5}$ doxycycline-induced cells were injected into the cerebellum of NOD/SCID mice (NOD.CB17-Prkdc scid $/ \mathrm{NCrCrl}$, Charles River, USA) through $0.5 \mathrm{~mm}$ burr hole in the midline, $2 \mathrm{~mm}$ posterior to lambda at $2 \mathrm{~mm}$ depth, using small animal stereotaxic frame under anesthesia [59]. Tumor growth was monitored by in vivo bioluminescence imaging using the IVIS Spectrum imaging system (Caliper Lifesciences, Perkin Elmer, MA, USA). Tumor bearing mice were maintained until they succumbed to the tumor or were about to succumb to the tumor as judged by over $40 \%$ loss of weight or other clinical symptoms. Upon sacrifice, whole brain was fixed in the neutral buffered formalin and embedded in a paraffin block. Hematoxylin \& Eosin stained sections of the paraffin blocks were used for determination of the invasive capacity of the tumor cells without revealing identity of the specimen to the analyst. 


\section{Transcriptome sequencing}

Libraries were prepared using the Truseq RNA sample prep kit V2 as per the manufacturer's protocol (Illumina, San Diego, USA) from the total RNA extracted from the medulloblastoma cells and subjected to 100 nucleotides deep sequencing using the Illumina HiSeq 2500 sequencing system to get a minimum of 10 million reads per library. The reads were aligned to the reference human genome hg19 using the TopHat version 2.0.13 (http:// ccb.jhu.edu/software/tophat) with default parameters. Raw counts for the reads aligned to the gene intervals were produced by the python package HTSeq version 0.6.1 (www-huber.embl.de/users/anders/HTSeq) using the default union-counting mode. The data was normalized by variance stabilizing transformation using the DESeq software that takes into account RNA-seq data size of each sample (http://bioconductor.org/packages/ release/bioc/html/DESeq.html). Gene Set enrichment analysis of the genes differentially expressed upon miR-204 expression was done using the GSEA (Gene Set Enrichment Analysis) software (software.broadinstitute. org/gsea/index.jsp). Downregulation of expression of known miR-204 target genes upon miR-204 expression in medulloblastoma cell lines was validated by SYBR green real time RT-PCR assay using gene-specific primers (Additional file 1: Table S1).

\section{Western blotting}

Total protein extracted from the medulloblastoma cells was separated by SDS-PAGE electrophoresis, blotted onto a PVDF membrane (Merck Millipore, Berlington, MA, USA) and probed with the primary antibody as per the manufacturer's protocol. The images were captured using the ChemiDoc gel imaging system (Biorad Hercules, CA, USA) or by autoradiography. The captured images were quantified using the Image Lab software (Bio-Rad, Hercules, CA, USA) or ImageJ software (imajeJ.nih.gov.in). The antibodies used for the Western blotting experiments are listed below.

I. Anti-LC3B (\#2775), anti-p62/SQSTM1 (\#8025), anti-Cathepsin D (\#2284), anti-Cathepsin B (\#31718) and, anti-IGF2R (\#14364) antibodies from the Cell signaling technology, Boston, MA, USA.

II. Anti-GAPDH antibody (SC 47724) from Santa Cruz Biotechnology, Dallas, TX, USA.

III. Anti-Histone H3 (acetyl K9) antibody (ab10812) from Abcam, Cambridge, UK.

\section{Luciferase reporter assay}

Firefly luciferase cDNA was cloned in the pcDNA 3.0 vector (Invitrogen, Carlsbad, CA, USA) downstream of the CMV promoter to generate 'pLuc' reporter vector. 3 '-UTR regions of the miR-204 target genes were amplified from the genomic DNA of normal human lymphocytes and cloned downstream of the firefly luciferase cDNA in the 'pLuc' vector. Putative miR-204 binding sites in the 3 '-UTRs were mutated by site-directed mutagenesis using primers having 4 nucleotides corresponding to the binding site altered [59]. Luciferase activity was assessed from the HEK293FT cells transfected with the luciferase reporter plasmid, miR-204 expressing plasmid/vector control pcDNA4 (Invitrogen, Carlsbad, CA, USA), and a plasmid vector expressing EGFP fluorescent protein. Luciferase activity was assessed from the total protein extracted from the transfected HEK293FT cells and was normalized against the EGFP fluorescence measured using the BioTek Cytation Hybrid Multimode Reader, Winooski, VT, USA.

\section{TRPM3/MIR-204 promoter methylation analysis and} upregulation of miR-204 expression upon treatment with histone deacetylase inhibitors

Genomic DNA was isolated from the medulloblastoma cell lines using QIAamp DNA mini kit (Qiagen, $\mathrm{GmbH}$, Hilden, Germany) as per the manufacturer's instructions. Bisulfite conversion of $500 \mathrm{ng}$ of the genomic DNA was performed using EZ DNA Methylation-Gold Kit from Zymo Research, Irvine, CA, USA, as per the manufacturer's instructions. The $203 \mathrm{bp}$ region covering - 200 to + 3 with respect to the known transcription start site of the TRPM3 gene was PCR amplified using the primers designed to amplify bisulfate converted DNA and sequenced (Additional file 1: Table S1). Medulloblastoma cells were treated with HDAC inhibitors Sodium valproate $(6 \mathrm{mM})$ and Trichostatin A ( $400 \mathrm{nM}$ ) for a period of $16 \mathrm{~h}$. Histone acetylation status was evaluated by separating total protein extracts from the treated cells by SDS-PAGE and probing the Western blot using anti-H3K9 acetylation antibody .

All experiments were performed at least three times and the Student's t-test was used for evaluating statistical significance of the difference in the test as compared to the control. Error bars indicate standard error of the mean/median.

\section{Results}

MiR-204 downregulation identifies a highly aggressive subset of Group 3 / Group 4 medulloblastomas having poor survival

MiR-204 expression was studied in an Indian cohort of 260 medulloblastomas and a non-overlapping MAGIC cohort of 763 medulloblastomas [2]. MiR-204 was found to be differentially expressed in the four molecular subgroups, with all WNT and $75-84 \%$ of Group 4 tumors having high miR-204 ( $R Q>20)$ while expression is low in almost all $\mathrm{SHH}$ subgroup tumors and in $54-76 \%$ of the Group 3 medulloblastomas (Fig. 1a, b). MiR-204 expression in the normal posterior fossa brain regions: cerebellum, mid-brain, pons and medulla, that are believed to be the sites of origin for the 4 molecular 

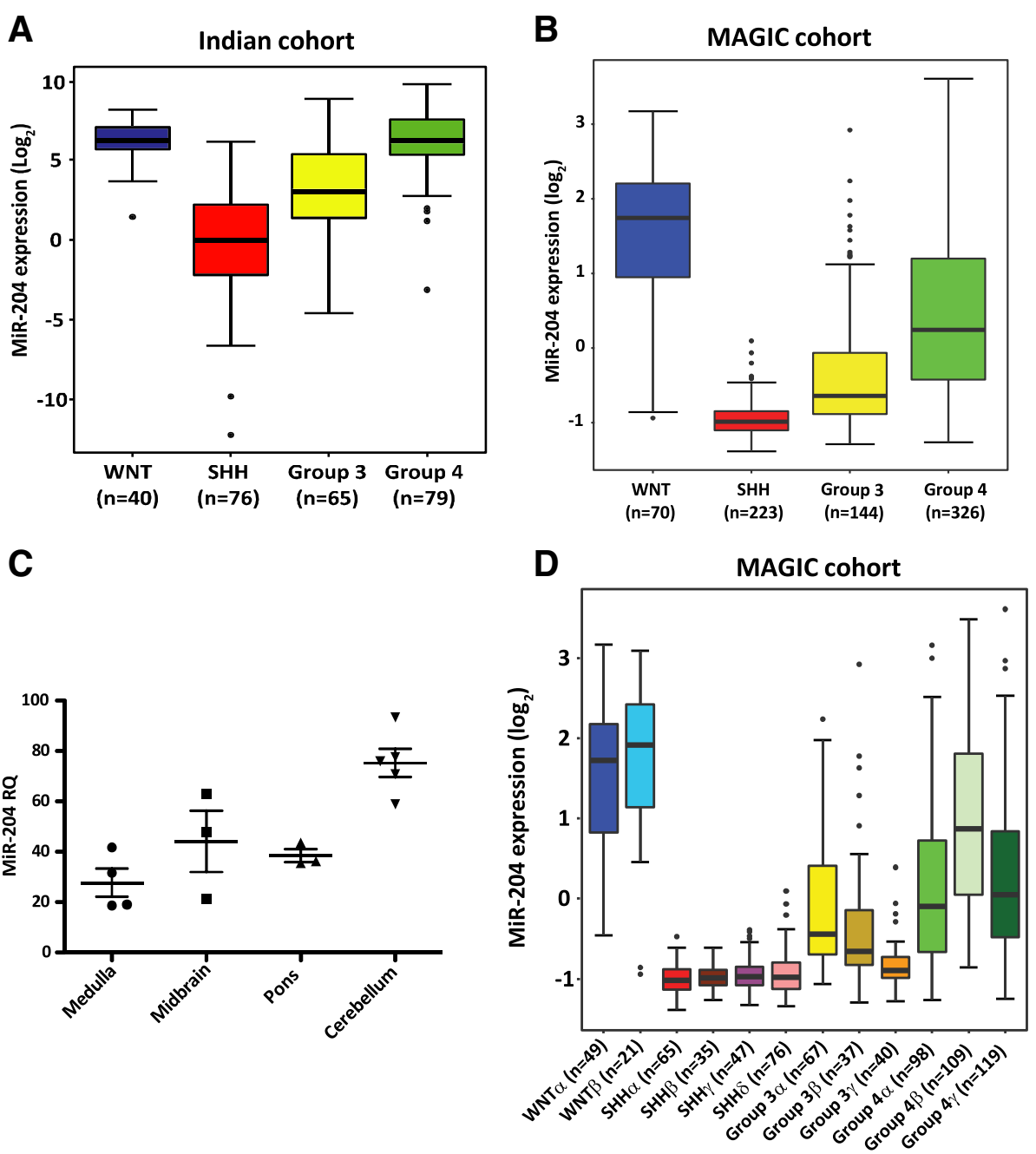

Fig. 1 MiR-204 expression across molecular subgroups/subtypes of medulloblastoma and in normal brain tissues. MiR-204 expression levels in the four subgroups of medulloblastomas from the Indian cohort $(\mathbf{a}, n=260)$ and the MAGIC cohort $(\mathbf{b}, n=763)$. $\mathbf{c}$. MiR-204 expression in the normal brain tissues from the posterior fossa region, evaluated by the Taqman assay. d. MiR-204 expression in the 12 subtypes of medulloblastomas from the MAGIC cohort $(n=763)$

subgroups of medulloblastoma [11], was found to range from $R Q=21$ to 93 (Fig. 1c). Thus, miR-204 expression is downregulated in the SHH subgroup and in a subset of the Group 3/Group 4 medulloblastomas. Integrated analysis of the genome wide DNA methylation data, expression data and copy number alterations data, has identified 12 subtypes corresponding to the four core subgroups of medulloblastomas [2]. MiR-204 expression levels were found to be high in both the WNT subtypes, low in all four SHH subtypes, low in all three Group 3 subtypes and low to moderate in two out of 3 subtypes of Group 4 (Fig. 1d). Group 3y having the worst outcome [2] has the least miR-204 expression among the Group 3 / Group 4 subtypes. Highly integrative analysis that integrated somatic mutation data analysis in addition to the genome wide methylation, transcriptome and copy number variation data has reported 8 subtypes within the Group 3/Group 4 medulloblastomas [34]. Analysis of MiR-204 expression in these 8 subtypes showed that the expression levels vary across the 8 subtypes with the least expression in the 3 subtypes (ii, iii and iv) that contain only Group 3 tumors (Additional file 2: Figure S1). The subtype ii enriched for MYC amplification has the least miR-204 expression levels. MYC amplification is a known marker for poor prognosis in the Group 3 medulloblastomas [44].

Group 3 / Group 4 medulloblastomas in the Indian cohort having metastasis at diagnosis were found to have significantly $(p=0.024)$ lower miR-204 expression (Fig. 2a). In the MAGIC cohort as well, miR-204 expression levels are lower in the Group 3 / Group 4 tumors having metastasis at diagnosis, although the difference is not 

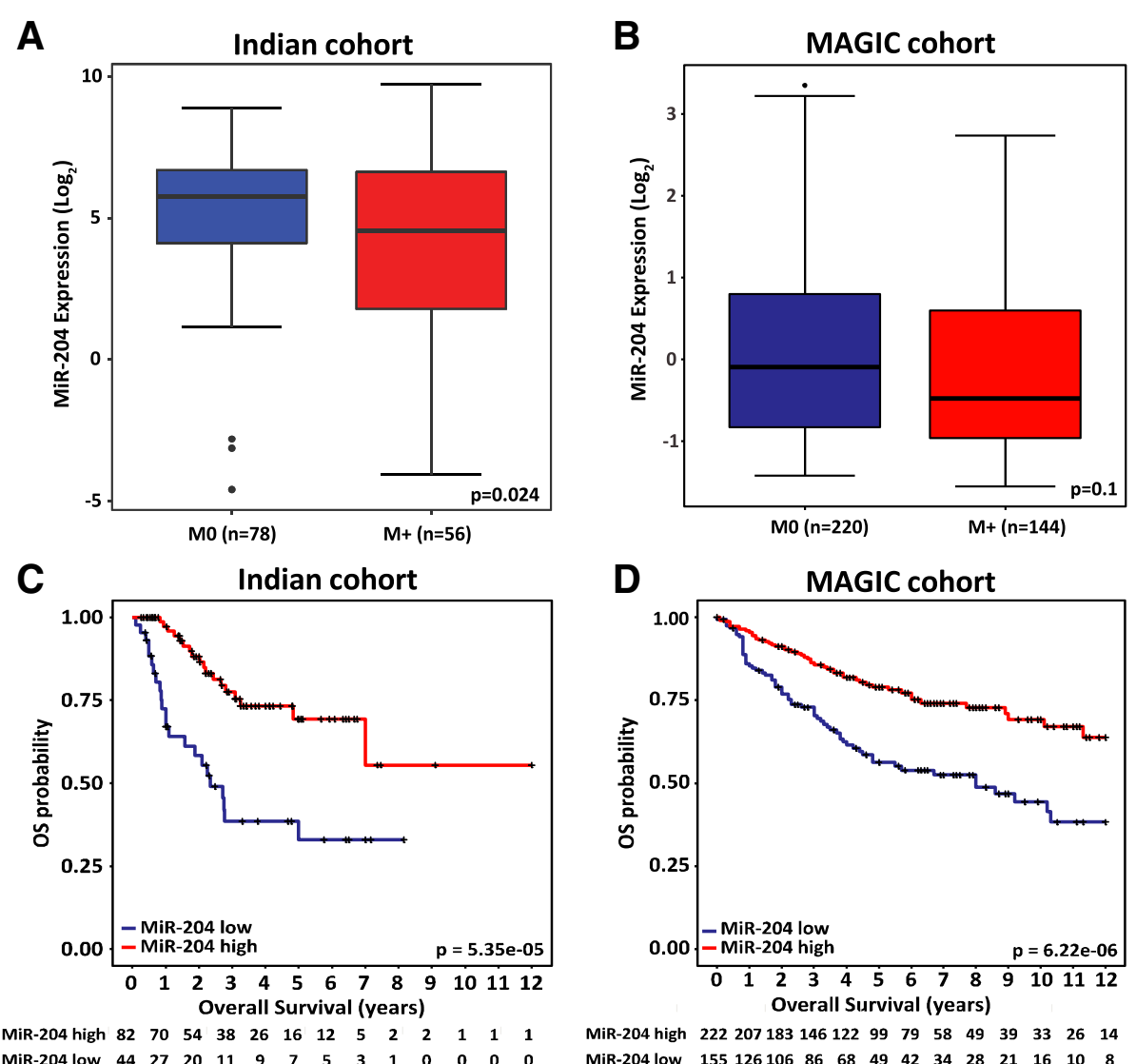

MiR-204 low $44 \begin{array}{llllllllllll}27 & 20 & 11 & 9 & 7 & 5 & 3 & 1 & 0 & 0 & 0 & 0\end{array}$
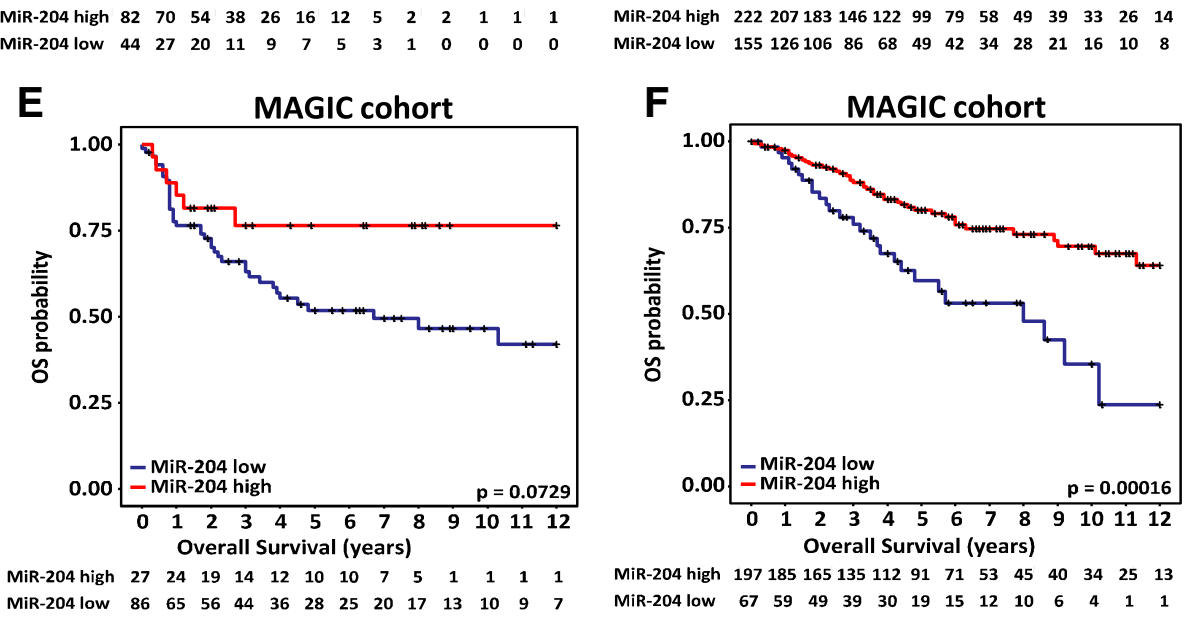

Fig. $\mathbf{2}$ Correlation of miR-204 expression with metastasis at diagnosis and overall survival. MiR-204 expression in Group 3 / Group 4 medulloblastomas having presence $(\mathrm{M}+$ ) or absence $(\mathrm{M} 0)$ of metastasis at diagnosis in the Indian cohort (a) and in the MAGIC cohort (b). Kaplan Meier survival analysis comparing overall survival of 'MiR-204 low' subset with that of 'MiR-204 high' subset of the Group 3 / Group 4 medulloblastomas from the Indian cohort (c) and from the MAGIC cohort (d). Kaplan Meier survival analysis comparing overall survival of 'MiR-204 low' with that of 'MiR-204 high' subset of Group 3 (e) and Group 4 (f) medulloblastomas from the MAGIC cohort

statistically significant (Fig. 2b). Low miR-204 expression in the Group 3 / Group 4 medulloblastomas was found to correlate with poor overall survival in the Indian cohort $(n=126)$ as well as in the larger MAGIC cohort $(n=377)$ (Fig. 2c, d). Five year overall survival of the 'miR-204 low' subset in the Indian cohort is $33 \%$ (95\% CI, 16.8-50.2\%) as compared to $69.4 \%$ (95\% CI, 54.1-80.4\%) of the 'miR-204 high' subset. In the MAGIC cohort as well, five year survival of the 'miR-204 low' subset is lower at 56.3\% (95\% CI $48.1 \%$ - 65. 9\%) as compared to $78.9 \%$ (95\% CI 73.1-85.2\%) of the 'miR-204 high' subset. Thus, low miR-204 levels identify a subset of Group 3/ Group 4 tumors having poor overall survival both in the Indian cohort and in the larger MAGIC cohort. 
In the MAGIC cohort within the Group 3 tumors, low expression levels of miR-204 have a trend towards worse survival, although it does not reach statistical significance due to lower fraction of 'miR-204 high' tumors (Fig. 2e). In the Indian cohort, low expression levels of miR-204 correlate with poor survival within the Group 3 as well (Additional file 3: Figure S2). Due to small proportion of 'miR-204 low subset' in the Group 4 tumors of the Indian cohort, survival analysis for the Group 4 was done only for the MAGIC cohort. The five year survival of the Group 4 'miR-204 high' subset is $80 \% \quad(95 \%$ CI 74-86.6\%) while that of the 'miR-204 low' subset is $59.7 \%(95 \%$ CI $47.2-75.4 \%)$ in the MAGIC cohort (Fig. 2f). Low miR-204 expression levels thus, identify a subset having poor overall survival within the Group 4 itself as well.

Restoration of miR-204 expression inhibits anchorageindependent growth, and tumorigenicity of medulloblastoma cells

MiR-204 expression levels in the established medulloblastoma cell lines D341, D425 and HD-MB03 were found to be in the range of $R Q=0.02$ to 0.14 (Fig. 3a). D341, D425 and the recently established HD-MB03 cell line belong to the Group 3 [16, 29]. MiR-204 expression in the D283 cell line which has characteristics intermediate between Group 3 and Group 4 is at $R Q=9.4 \pm 1.0$ (Fig. 3a) [16]. The cell lines were transduced with the pTRIPZ lentiviral vector expressing miR-204 in a doxycycline inducible manner. Stable polyclonal populations of the four medulloblastoma cell lines express miR-204 at levels ( $R Q=25$ to 70 ) comparable to that in the normal brain tissues after induction with doxycycline (Fig. 3a). Effect of miR-204 expression on the proliferation and anchorage-independent growth of these cell lines was studied by the MTT assay and soft agar colony formation assay respectively. While miR-204 expression inhibited proliferation of D283 and D425 cells by 25 to $40 \%$, it did not affect proliferation of D341 and HD-MB03 medulloblastoma cells (Fig. 3b). MiR-204 expression resulted in significant inhibition of soft agar colony formation capacity ( 35 to $55 \%, p<0.001$ ) of all the four medulloblastoma cell lines studied (Fig. 3c, d).

In order to study the effect of miR-204 expression on tumorigenic potential, D283, D341, HD-MB03 cells as

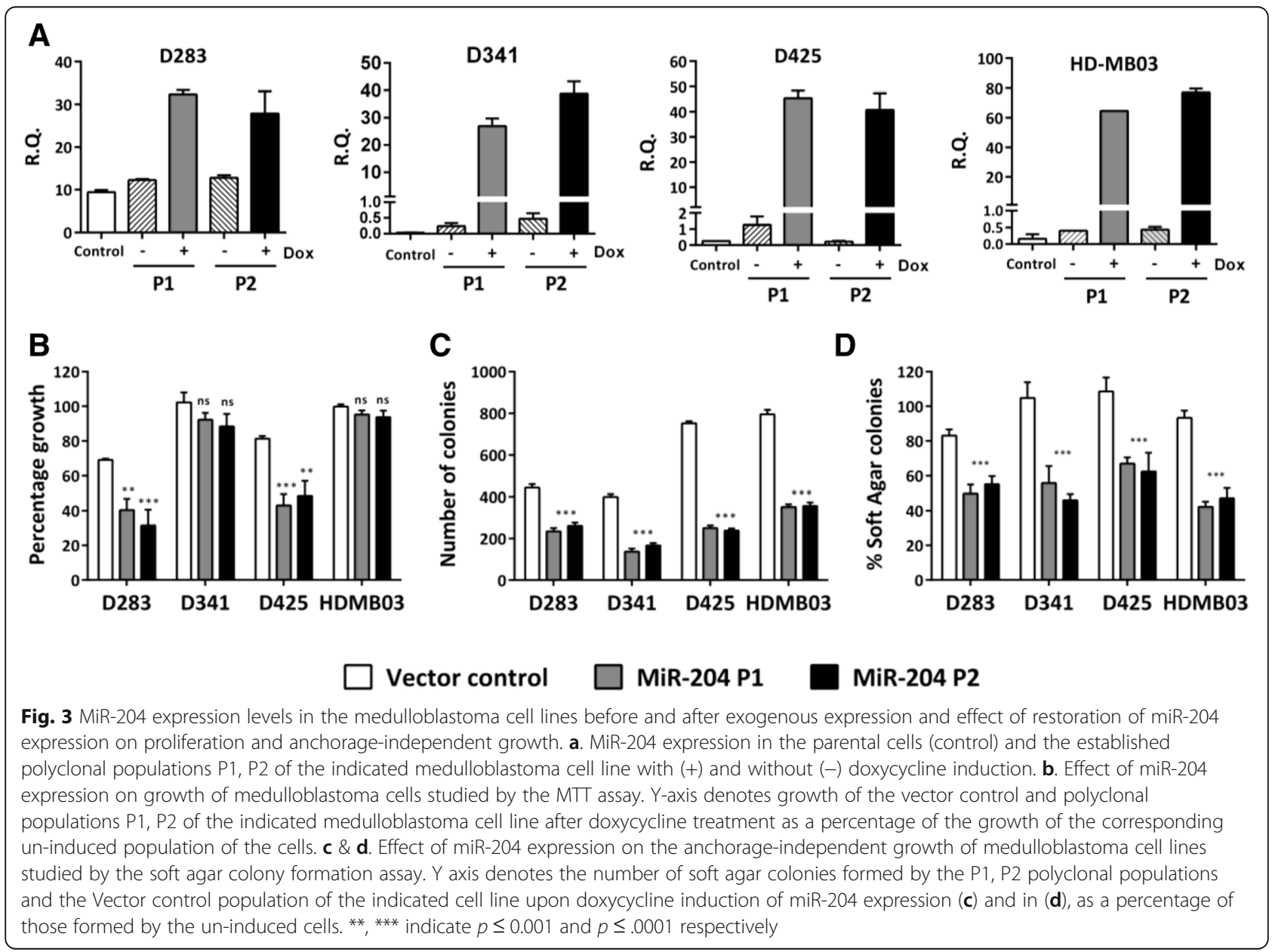


well as their miR-204 expressing polyclonal population cells were engineered to express firefly luciferase and, were injected stereotactically in cerebellum of NOD/ SCID mice after doxycycline induction. MiR-204 expression was found to significantly $(p<0.002$ to 0.0001$)$ decrease tumorigenicity of all the 3 medulloblastoma cell lines as judged by the in vivo imaging of the orthotopic tumors (Fig. 4a). The tumor volume decreased by 8.8-fold to 25-fold upon miR-204 expression (Fig. 4b). Further, survival of the tumor bearing mice increased by 26 to 34\% upon miR-204 expression (Fig. 4c) in all the three medulloblastoma cell lines studied.

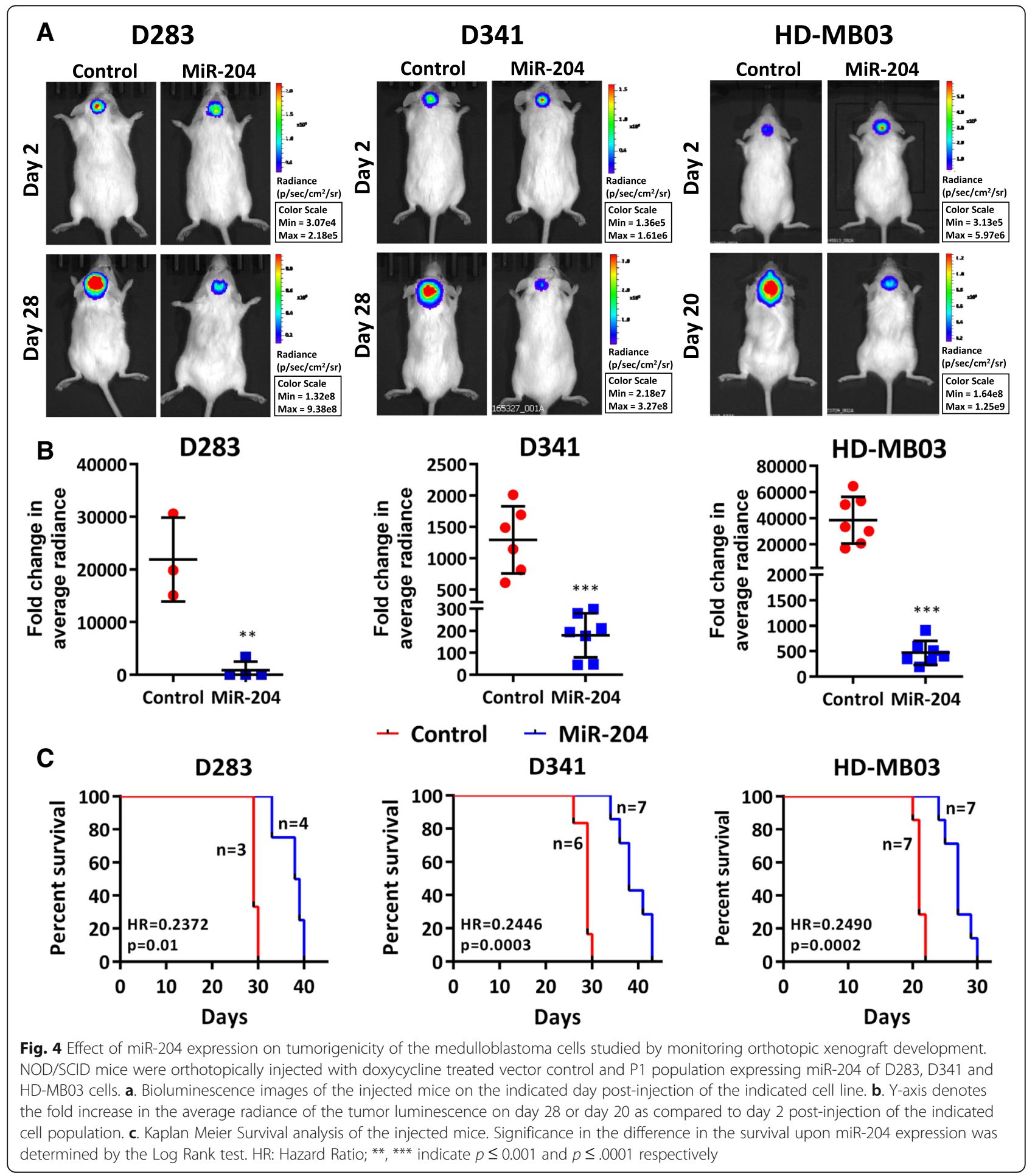


MiR-204 expression inhibits invasion potential of medulloblastoma cells in vitro and in vivo

Effect of miR-204 expression on the invasion potential of medulloblastoma cells was studied by evaluating invasion of the cells through Matrigel ${ }^{\mathrm{TM}}$ coated membranes in transwell inserts. Figure 5a shows images of the Calcein-AM labeled medulloblastoma cells that have invaded the matrigel coated membrane after $56 \mathrm{~h}$ to 72 h. Evaluation of the fluorescence intensity of the invaded cells showed $60-80 \%$ reduction in the invasion potential of D283 and HD-MB03 cells upon miR-204 expression (Fig. 5b). Furthermore, invasive capacity of the medulloblastoma cells as judged by their in vivo invasion across the cerebellar folia boundary was found to be reduced upon miR-204 expression
(Fig. 5c, d). Thus, miR-204 expression reduced invasion potential of the medulloblastoma cells both in vitro and in vivo.

\section{MiR-204 expression downregulates M6PR, IGF2R genes involved in the lysosomal pathway and inhibits autophagy of medulloblastoma cells}

In order to delineate the molecular mechanism underlying the tumor suppressive role of miR-204 in medulloblastoma cells, genes significantly differentially expressed upon miR-204 expression were identified by the RNA-seq analysis. Figure 6a shows a heat map of the top 60 genes downregulated upon miR-204 expression in HD-MB03 cells. GSEA analysis identified most significant enrichment of Epithelial Mesenchymal Transition

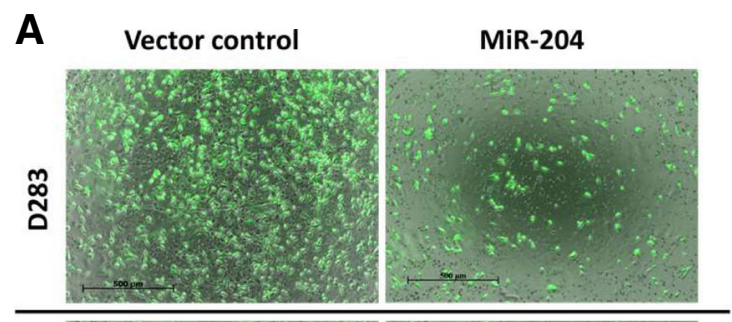

B
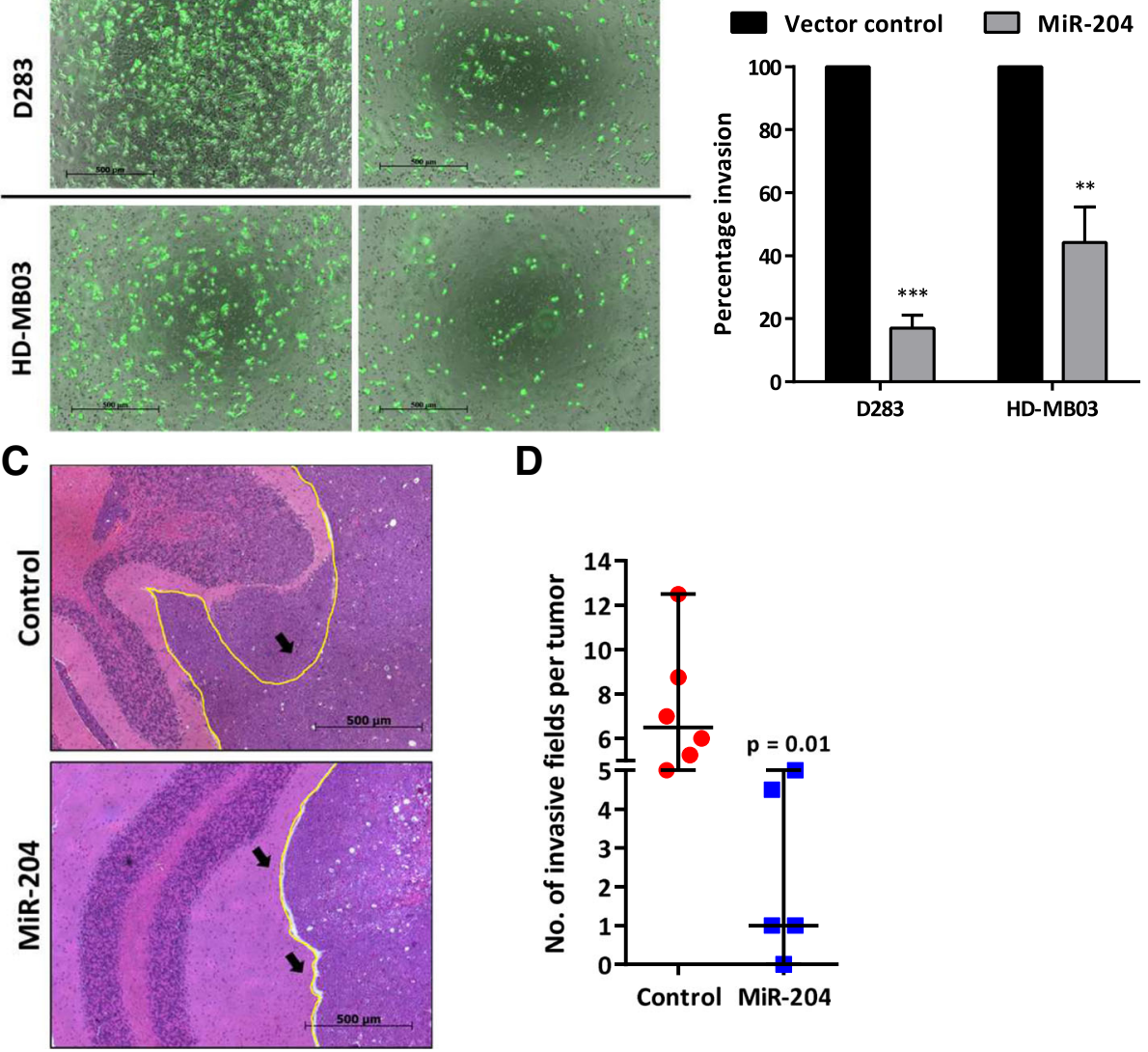

Fig. 5 Effect of miR-204 expression on in vitro and in vivo invasion potential of medulloblastoma cells. a. Representative Images of the Calceinlabeled cells on the lower side of the transwell insert membrane, post-invasion of the doxycycline treated miR-204 expressing or vector control polyclonal population of D283 and HD-MB03 cells. b. Y- axis indicates the percentage of the invaded cells of the miR-204 expressing polyclonal population as compared to the Vector Control population of the cells, evaluated by measuring fluorescence intensity of the invaded cells. $\mathbf{c}$. Photographs of hematoxylin-eosin stained paraffin sections (sagittal section) of the orthotopic xenografts of doxycycline treated vector control cells and miR-204 expressing polyclonal population of D341 cells. Arrows show the vector control cells invading cerebellar folia boundary as compared to the cohesive margin of the miR-204 expressing cells. Yellow line indicates cerebellar folia margin. $\mathbf{d}$. The scatter dot plot shows significant difference in the number of invasive fields per tumor in the miR-204 expressing tumors as compared to those expressing vector control D341 cells. ${ }^{* *},{ }^{* *}$ indicate $p \leq 0.001$ and $p \leq .0001$ respectively 

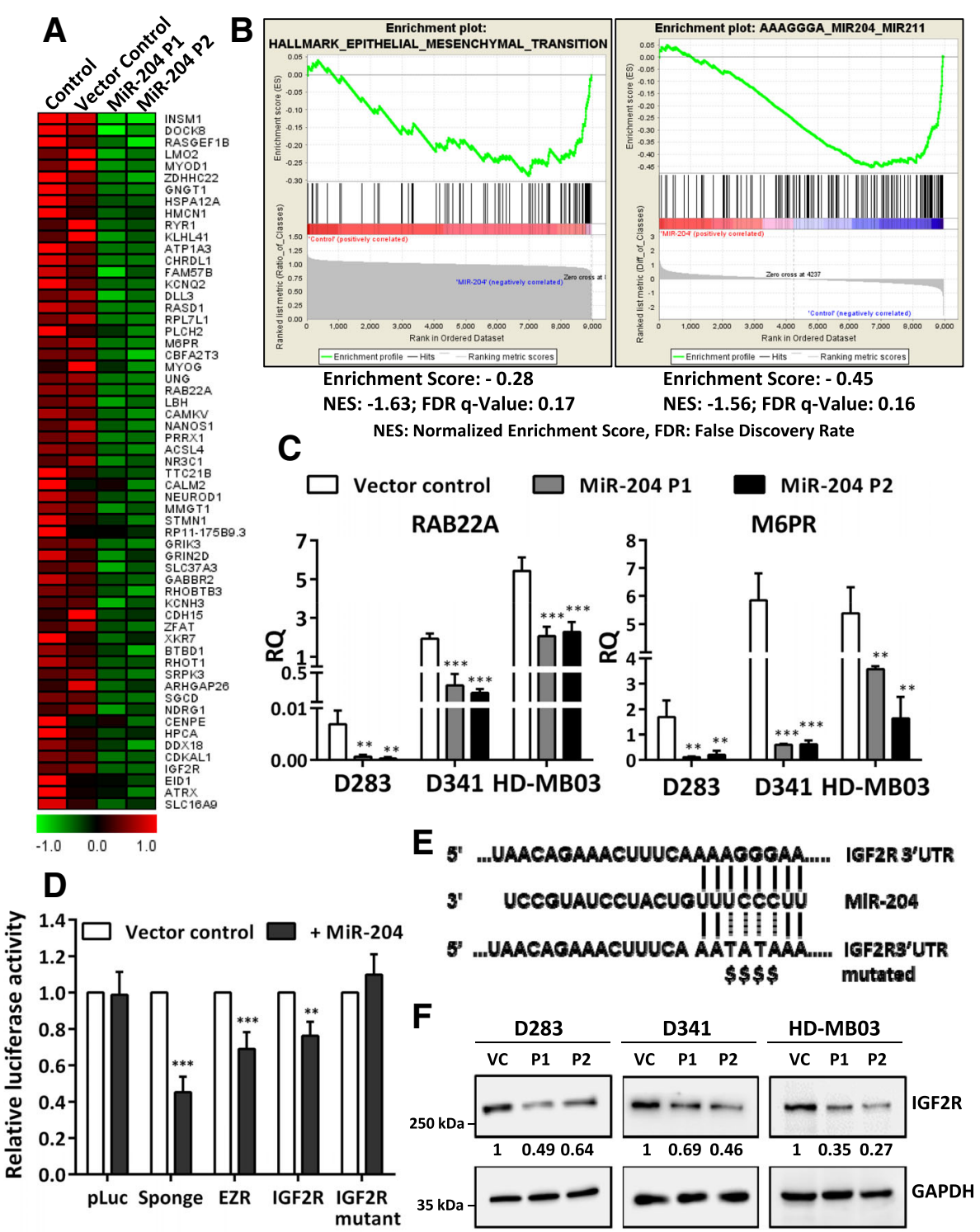

Fig. 6 Downregulation of miR-204 target genes upon its expression in medulloblastoma cells. a. Heat map shows top 60 rank ordered genes (GSEA) whose expression is downregulated upon miR-204 expression in the two polyclonal populations, as compared to the doxycycline treated parental HD-MB03 cells and the vector control cells, identified by the RNA-seq analysis. Known validated miR-204 targets M6PR and RAB22A are indicated by arrows. b. GSEA analysis of the genes downregulated upon miR-204 expression in HD-MB03 cells showed most significant enrichment of the Epithelial Mesenchymal transition genes and the 'miR-204 target gene set' (software.broadinstitute.org/gsea/msigdb/ collections.jsp). c. Real time RT-PCR analysis showing downregulation of the known miR-204 targets RAB22A, M6PR upon miR-204 expression in the medulloblastoma cell lines. $\mathbf{d}$. $Y$ axis shows luciferase activity of the indicated p-Luc construct upon co-transfection with miR-204 expressing plasmid relative to that obtained upon cotransfection with the Vector control. 3'-UTR of the EZR gene and a sponge construct containing six miR204 binding sites were used as positive controls. e Nucleotide sequence of the miR-204 target site in the $3^{\prime} U T R$ of the IGF2R gene and the mutations introduced indicated by '\$'. f. Western blot analysis showing downregulation of IGF2R protein levels upon miR-204 expression in the polyclonal populations P1, P2 of the indicated medulloblastoma cell line as compared to the doxycycline treated vector control (VC) cells. The numbers below the western blot indicate fold change in the IGF2R protein levels upon miR-204 expression level using GAPDH levels as a housekeeping control for normalization. ${ }^{* *},{ }^{* * *}$ indicate $p \leq 0.001$ and $p \leq .0001$ respectively

genes in the genes downregulated upon miR-204 expression (Fig. 6b). GSEA analysis using the microRNA target motif database identified most significant enrichment of miR-204 targets in the genes downregulated upon miR-204 expression (Fig. 6b). Known validated targets of
miR-204 like $R A B 22 A, M 6 P R$ are at the top of the list whose downregulation upon miR-204 expression was further confirmed by real time RT-PCR in the three medulloblastoma cell lines (Fig. 6c). IGF2R was one of the putative miR-204 targets identified by the GSEA analysis. 3'-UTR 
regions of $I G F 2 R$ and the known target $E Z R$ were cloned downstream of the luciferase cDNA in the pcDNA3.0 vector. Luciferase reporter assay showed inhibition of luciferase activity upon co-transfection of these $3^{\prime}$-UTR constructs with the vector expressing miR-204 in HEK293FT cells suggesting IGF2R as a direct target of miR-204 (Fig. 6d). MiR-204 mediated inhibition of the luciferase activity was lost upon site-directed mutagenesis of the miR-204 binding site in the 3 '-UTR of IGF2R, validating it as a direct target of miR-204 (Fig. 6d, e). Furthermore, downregulation of IGF2R protein levels upon miR-204 expression was confirmed by the western blotting in all the three medulloblastoma cell lines (Fig. 6f).

Both cation-dependent and cation-independent man nose-6-phosphate receptors i.e. M6PR and IGF2R respectively are known to be involved in trafficking of lysosomal proteases from the Golgi apparatus to lysosomes [26]. Therefore, effect of miR-204 expression on the levels of lysosomal enzymes Cathepsin B and Cathepsin D in medulloblastoma cells was studied by western blotting. MiR-204 expression resulted in considerable downregulation of these lysosomal enzymes in all three medulloblastoma cell lines D283, D341 and HD-MB03 (Fig. 7a). Lysosomal degradation pathway plays a major role in autophagy [12]. Besides, LC3B is a known target of miR-204 that plays a crucial role in autophagy [28]. Effect of miR-204 expression on autophagy was studied by evaluating LC3B flux. Upon autophagy induction, LC3BI isoform gets converted to LC3BII as a result of conjugation with phosphatidylethanolamine [12]. LC3BII levels however, decrease upon fusion of autophagosome to lysosome due to degradation by lysosomal enzymes. LC3B turnover is therefore studied in the presence and absence of an inhibitor of lysosomal degradation like chloroquine to evaluate LC3B flux [18]. MiR-204 expression resulted in high LC3BI / LC3BII ratio in D283 cells both before and after chloroquine treatment indicating low LC3B flux and thereby autophagy inhibition (Fig. 7b). In D341 and HD-MB03 medulloblastoma cells, total levels of LC3B decrease upon miR-204 expression both before and after treatment with chloroquine, indicating lower LC3B turnover and thereby autophagy inhibition (Fig. 7b). Autophagy inhibition is
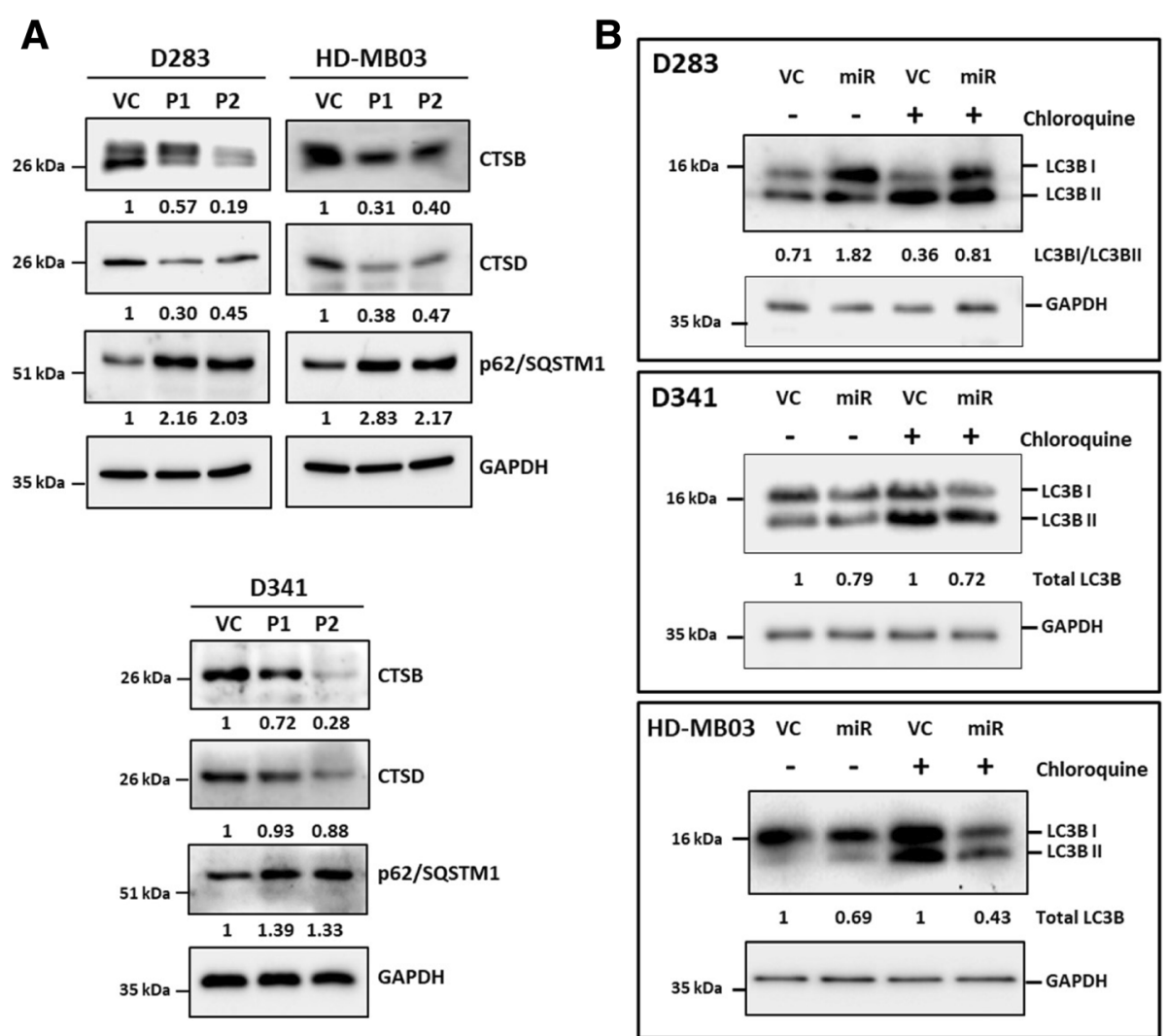

Fig. 7 Effect of miR-204 expression on lysosomal enzymes and autophagy in medulloblastoma cells. a. Western blot analysis showing reduction in the levels of Cathepsin B (CTSB), Cathepsin D (CTSD) and increase in the levels of p62/SQSTM1 in the P1, P2 polyclonal populations of D283, D341 and HD-MB03 cells upon miR-204 expression. b. LC3BI, LC3BII levels in the miR-204 expressing polyclonal populations of D283, D341 and HD-MB03 cells as compared to the doxycycline treated vector control (VC) cells before and after treatment with Chloroquine for $1 \mathrm{~h}$. The numbers below the western blots indicate the fold change in the indicated protein level or LC3BI / LC3B II ratio upon miR-204 expression using GAPDH as a house-keeping control 
known to be accompanied by increase in the levels of p62/ SQSTM1 adapter protein [12]. Expression levels of p62/ SQSTM1 increased upon miR-204 expression in the three medulloblastoma cell lines further confirming autophagy inhibition (Fig. 7a). Thus, miR-204 expression inhibits autophagy mediated degradation pathway in medulloblastoma cells.

\section{TRPM3/MIR204 promoter methylation analysis and} upregulation of miR-204 expression upon treatment with HDAC inhibitors

MiR-204 is located within the cancer associated genomic region at 9q21.1-q22.3 that exhibits high frequency of loss of heterozygosity in various cancers [55]. Loss of chromosome 9q is however, not frequent in Group 3 / Group 4 medulloblastomas with less than $20 \%$ and $5 \%$ loss of chr $9 q$ arm in Group 3, Group 4 tumors respectively based on the analysis of structural variations in 1000 medulloblastomas [38]. Downregulation of miR-204 expression has also been reported to occur as a result of promoter methylation [58]. CpG island at the TRPM3/MIR204 promoter locus seems to be not methylated in Group 3 / Group 4 medulloblastomas based on the data from the Illumina $450 \mathrm{~K}$ array and bisulfite sequence analysis done on Group 3 medulloblastoma cell lines for the CpG island in the TRPM3/MIR204 promoter region (Fig. 8a; Additional file 4: Figure S3). HDAC inhibitors have been reported to inhibit growth of medulloblastoma cells particularly that of $M Y C$-driven
Group 3 cell lines [29, 43]. Treatment of medulloblastoma cell lines D283, D425 and HD-MB03 with Trichostatin A and Sodium valproate, the HDAC inhibitors resulted in 2 to 4 fold increase in expression levels of miR-204 (Fig. 8b) accompanied by the increased histone acetylation (Fig. 8c). Thus, treatment of medulloblastoma patients with HDAC inhibitors could help in upregulation of miR-204 that has tumor suppressive effect.

\section{Discussion}

In a large scale study on 3312 tumors and 1107 non-malignant tissues contributed by 51 different cancer types, miR-204-211 family was found to be the top deleted microRNA family in cancer, suggesting its crucial role as a tumor suppressive miRNA in multiple cancer types [55]. In the present study, miR-204 was found to be differentially expressed in the four core molecular subgroups of medulloblastomas, with almost all $\mathrm{SHH}$ and a subset of Group 3/Group 4 tumors showing downregulation of miR-204 expression. Despite the complexity of the heterogeneity and overlap present in the copy number variations, methylation profiles and somatic mutation profiles in the Group 3 / Group 4 medulloblastomas [2], miR-204 expression levels identify a subset of these tumors having poor survival in the Indian as well as in the large MAGIC cohort. This finding is consistent with lower expression of miR-204 correlating with poor survival in breast cancer [23], non-small

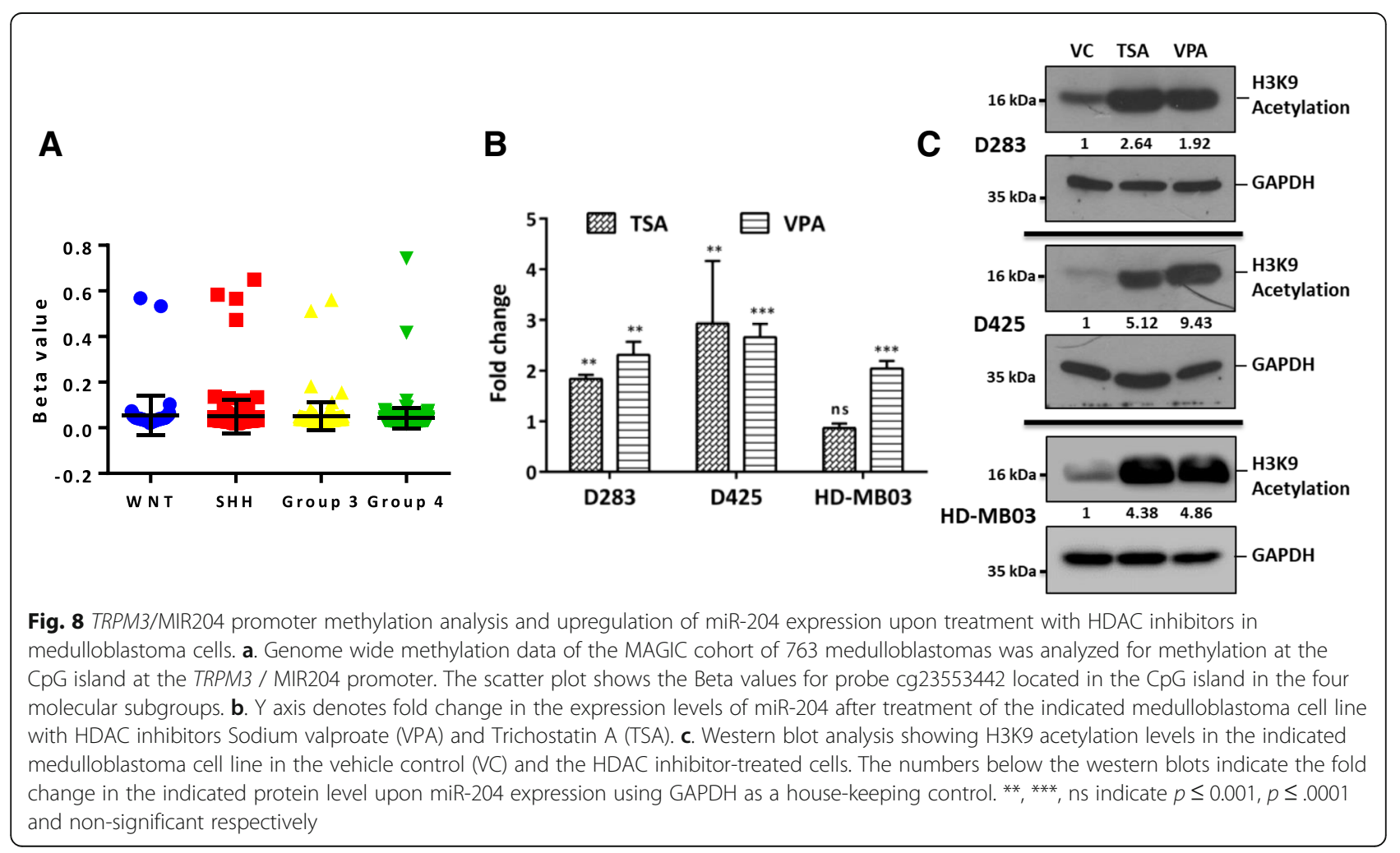


cell lung cancer [15], and neuroblastoma [47]. Integrated genomic studies have identified novel molecular subtypes within the four core subgroups of medulloblastomas $[2,49]$. Among the three Group 3 subtypes, subtype $3 \gamma$ was found to have the worst 5 year survival rate of $41.9 \%$ as compared to that of subtype $3 \alpha$ and subtype $3 \beta$ at 66.2 and $55.8 \%$ respectively [2]. Group $3 \gamma$ having the worst survival showed the least miR-204 expression among the 3 subtypes of Group 3. The Group 4 subtypes do not show significant difference in their overall survival [2]. MiR-204 expression on the other hand, identified a subset of Group 4 medulloblastomas having significantly poor survival of $59.7 \%$ as compared to $80 \%$ of the 'miR-204 high' subset. Group 3 poor prognostication markers like $M Y C$ amplification and isochrome $17 \mathrm{q}$ do not have prognostication value in Group 4 [44]. FSTL5 immunopositivity serves as a marker for poor prognostication in both Group 3 and Group 4 medulloblastomas [45]. Adult Group 4 patients have also been reported to have poor survival rates [46]. Loss of chromosome 11 or gain of chromosome 17 identify a small subset of Group 4 patients who have excellent survival [51]. Biology underlying these cytogenetic alterations is however, not understood. Thus, low miR-204 expression serves as a marker of poor prognosis in Group 4 that has paucity of markers for prognostication. Integrated genomic analysis is expensive as well as technically demanding and thus cannot be used in routine clinical practice for risk stratification. MiR-204, a single microRNA on the other hand, can be easily combined with the Nanostring assay that classifies medulloblastomas into the four molecular subgroups [39]. Furthermore, miR-204 due its small size resists degradation during formalin fixation and thus would be a reliable marker even in poor quality FFPE tissues.

Downregulation of miR-204 expression with poor survival is consistent with its tumor-suppressive effect in medulloblastoma cell lines. Restoration of miR-204 expression in multiple established Group 3 medulloblastoma cell lines was found to inhibit their anchorage-independent growth, invasion potential and tumorigenicity. Tumor suppressive effect of miR-204 in the MYC amplified Group 3 cell lines is remarkable since other microRNAs downregulated in medulloblastoma like miR-206 for instance, fail to inhibit tumorigenicity of these cell lines [41]. MiR-204 has been shown to inhibit invasion and tumorigenicity of various cancer cells including glioma, colorectal cancer, endometrial cancer and cervical cancer cells [3, 27, 57, 58]. Thus, the tumor suppressive role of miR-204 in medulloblastoma cells is consistent with its role in other cancers.

MiR-204 has been reported to target a number of genes including RAB22A, FOXC1, EZR, BCL2L2, M6PR, BCL2, MCL1, FOXA1, FOXM1, EPHB2 [22, 42, 50, 52, 57]. Transcriptome sequencing / real time RT-PCR / western blot analysis showed downregulation of RAB22A, M6PR, EZR, $E P H B 2$, upon miR-204 expression in medulloblastoma cells as well. $I G F 2 R$ was identified and validated as a novel target of miR-204. MiR-204 expression in medulloblastoma cells resulted in downregulation of both M6PR and IGF2R that mediate transport of lysosomal enzymes from the Golgi apparatus to lysosomes [26]. Furthermore, reduction in the levels of lysosomal enzymes Cathepsin B and Cathepsin D upon miR-204 expression in medulloblastoma cells suggests impairment of the lysosomal degradation pathway. Autophagy brings about p62/SQSTM1 mediated degradation of its cargo by lysosomal degradation pathway [12] MiR-204 is known to target LC3B, a crucial mediator of autophagy [28]. In the present study as well, miR-204 expression in medulloblastoma cells resulted in reduction in the LC3B flux and increase in the levels of p62/SQSTM1 indicating autophagy inhibition. Autophagy has been shown to play role in tumor promotion by sustaining survival in stress, by reducing oxidative stress and, maintaining metabolic homeostasis [14]. Inhibition of tumor growth upon miR-204 expression is consistent with these reports on the role of autophagy in tumor promotion. Autophagy has also been reported to promote invasion by activating Epithelial Mesenchymal Transition of hepatocellular carcinoma cells [48], by promoting secretion of factors like IL6, MMP2 [24] and by activating the MAP kinase signaling pathway in glioblastoma cells [8]. Consistent with the inhibition of invasion capacity of medulloblastoma cells upon miR-204 expression, downregulation of miR-204 expression was found to be associated with higher incidence of metastasis at diagnosis in Group 3 / Group 4 medulloblastomas. Thus, poor survival of Group 3 / Group 4 medulloblastomas having low miR-204 expression is likely due to their higher invasive capacity and higher malignant potential.

Several microRNAs whose expression is deregulated in medulloblastoma are known to play role in embryonic brain development [56]. MiR-9 and miR-124a that play crucial role in the onset of neurogenesis by targeting transcription factors like SOX9, FOXG1 and MEIS1, are downregulated in medulloblastoma [6]. MiR-9 and miR-199b-5p target HES1, thereby silence Notch signaling pathway at the onset of neuronal differentiation [7, 10]. Low expression of miR-9 and miR-199b-5p has been found to correlate with poor survival in medulloblastoma and their expression in medulloblastoma cell lines promotes growth arrest [7, 10]. MiR-17-92 cluster microRNAs are overexpressed predominantly in the SHH subgroup medulloblastomas [35]. Knock-out of this microRNA cluster brings about reduction in size of cerebellum and inhibits medulloblastoma formation in Ptch knock-out mouse model of SHH subgroup medulloblastomas indicating role of these microRNAs in normal development and tumorigenesis [33]. MiR-204 has been reported to play crucial role in lens and retinal 
development by targeting MEIS2 transcription factor in Medaka fish [4]. MiR-204 expression has been found to be upregulated during aging in mouse hippocampus and target Ephrin B2 that plays role in axon guidance [31]. MiR-204 has also been reported to control neuronal migration and cortical morphogenesis in mouse embryos presumably by targeting Doublecortin that is known to play role in neuronal migration [54]. Effect of miR-204 on invasive capacity of medulloblastoma cells is consistent with the role of miR-204 in neuronal migration. Thus, miR-204 appears to play role in both normal brain development and tumorigenesis like several other miRNAs that are known to be deregulated in medulloblastoma.

Delineating the molecular mechanism underlying downregulation of miR-204 expression would suggest ways to increase its expression, thereby improving survival rate of medulloblastoma patients. Group 3 medulloblastoma cells treated with HDAC inhibitors showed modest 2 to 4 fold increase in the miR-204 expression levels. Treatment with HDAC inhibitors has been reported to inhibit medulloblastoma cell growth in several studies [20, 29, 43]. Thus, HDAC inhibitors appear to have therapeutic potential in the treatment of medulloblastoma.

\section{Conclusions}

In summary, downregulation of miR-204 expression correlates with poor survival in the Group 3 / Group 4 medulloblastomas. Furthermore, within the Group 4 itself, low expression of miR-204 identifies a subset having significantly poor survival, making it a valuable marker for risk stratification in the subgroup that has paucity of prognostication markers. Restoration of miR-204 expression leading to reduction in the invasive capacity and tumorigenic potential of medulloblastoma cells suggests therapeutic potential of miR-204 in the treatment of medulloblastomas. Upregulation of miR-204 expression upon treatment with HDAC inhibitors, although modest, suggests a role of these inhibitors in the treatment of medulloblastomas.

\section{Additional files}

Additional file 1: Table S1. The nucleotide sequences of the primers used in the study. All sequences are given in $5^{\prime}$ to $3^{\prime}$ direction. (DOCX $15 \mathrm{~kb}$ ) Additional file 2: Figure S1. MiR-204 expression levels in the 8 subtypes of Group 3 / Group 4 medulloblastomas from Northcott et al. [34] data. (PPTX $39 \mathrm{~kb}$ )

Additional file 3: Figure S2. Kaplan Meier Survival Analysis of Group 3 medulloblastomas from the Indian cohort comparing overall survival of 'miR-204 high' subset with that of 'miR-204 low' subset. (PPTX 69 kb)

Additional file 4: Figure S3. Methylation analysis of the CpG island from the promoter region of TRPM3/MIR204. A 203 bp region of the CpG island from the promoter region of the TRPM3 gene was PCR amplified from bisulfite converted genomic DNA of the medulloblastoma cell lines. Representative nucleotide sequence of this PCR product from the indicated medulloblastoma cell line is shown. Arrows indicate the CpG residues in the DNA sequence and their sequence in the bisulfite converted (BSP) DNA from the medulloblastoma cells. (PPTX $121 \mathrm{~kb}$ )

\section{Abbreviations}

3'-UTR: 3'- untranslated region; ANOVA: Analysis of variance; ATCC: American Type Culture Collection; Cl: Confidence interval; DMEM: Dulbecco's Modified Eagle Medium; FBS: Fetal Bovine Serum; FFPE: Formalin- Fixed, ParaffinEmbedded; GSEA: Gene Set Enrichment Analysis; HDAC: Histone deacetylase; MAGIC: Medulloblastoma Advanced Genomics International Consortium; RMA: Robust Multi-array Average; RQ: Relative Quantity; RT-PCR: Reverse Transcription-Polymerase Chain Reaction; SDS-PAGE: Sodium Dodecyl Sulphate-Polyacrylamide Gel Electrophoresis; STR: Short Tandem Repeat

\section{Acknowledgements}

We thank Mr. Anant Sawant for technical assistance and Ms. Nazia Bano, Ms. Amita Wawdekar, clinical trial coordinators. We thank Prof. S. K. Shankar, NIMHANS, Bengaluru for making normal brain tissues available for the study.

\section{Funding}

We thank Department of Biotechnology, India for the financial support. Dr. V. Ramaswamy is supported by operating grants from the Canadian Institutes for Health Research, the Brain Tumor Foundation of Canada, Meagan's Walk and the American Brain Tumor Association.

\section{Availability of data and materials}

The datasets used during the current study are available from the corresponding author on reasonable request.

\section{Authors' contributions}

$H B, R P$ and PP have done the major experimental work involving Group 3 medulloblastoma cell lines. AD, SK, RK, KY have contributed to the experimental work that includes MicroRNA profile, molecular classification of medulloblastomas and evaluation of tumorigenicity. SM and Nikhil G have done the transcriptome sequencing and bioinformatics analysis. RJ, ES, TG, AM, AG, NG, PS, GC have contributed by recruitment of medulloblastoma cases, diagnosis and clinical data including treatment given and follow-up. NVS has contributed to the experimental design, implementation, analysis and, interpretation. VR has contributed to the Experimental design, analysis and interpretation. All authors read and approved the final manuscript.

\section{Ethics approval and consent to participate}

The study was approved by the Institutional Ethics Committee of the Tata Memorial Centre. Tumor tissues were procured after getting informed consent from the patients. Animal study was approved by the Institutional Animal Ethics Committee.

\section{Consent for publication}

Not applicable.

\section{Competing interests}

The authors declare that they have no competing interests.

\section{Publisher's Note}

Springer Nature remains neutral with regard to jurisdictional claims in published maps and institutional affiliations.

\section{Author details}

${ }^{1}$ Shirsat Laboratory, Advanced Centre for Treatment, Research \& Education in Cancer, Tata Memorial Centre, Kharghar, Navi Mumbai 410210, India.

${ }^{2}$ Department of Radiation Oncology, Tata Memorial Centre, Kharghar, Navi Mumbai 410210, India. ${ }^{3}$ Bioinformatics Centre, Advanced Centre for Treatment, Research \& Education in Cancer, Tata Memorial Centre, Kharghar, Navi Mumbai 410210, India. ${ }^{4}$ Department of Radiation Oncology, Tata Memorial Hospital, Tata Memorial Centre, Parel, Mumbai 400012, India. ${ }^{5}$ Department of Pathology, Tata Memorial Hospital, Tata Memorial Centre, Parel, Mumbai 400012, India. ${ }^{6}$ Department of Surgical Oncology, Tata 
Memorial Hospital, Tata Memorial Centre, Parel, Mumbai 400012, India. ${ }^{7}$ Department of Medical Oncology, Tata Memorial Hospital, Tata Memorial Centre, Parel, Mumbai 400012, India. ${ }^{8}$ Department of Neurosurgery, Seth G. S. Medical College \& K. E. M. Hospital, Parel, Mumbai 400012, India. ${ }^{9}$ Department of Pathology, Seth G. S. Medical College \& K. E. M. Hospital, Parel, Mumbai 400012, India. ${ }^{10} \mathrm{Homi}$ Bhabha National Institute, Training School Complex, Anushakti Nagar, Mumbai 400085, India. ${ }^{11}$ Division of Haematology/Oncology, Department of Paediatrics, Hospital for Sick Children and University of Toronto, 555 University Ave, Toronto, ON M5G 1X8, Canada.

\section{Received: 30 January 2019 Accepted: 10 March 2019} Published online: 03 April 2019

\section{References}

1. Bourdeaut F, Miquel C, Alapetite C, Roujeau T, Doz F (2011) Medulloblastomas: update on a heterogeneous disease. Curr Opin Oncol 23: 630-637

2. Cavalli FMG, Remke M, Rampasek L, Peacock J, Shih DJH, Luu B et al (2017) Intertumoral heterogeneity within Medulloblastoma subgroups. Cancer Cell 31:737-754 e736. https://doi.org/10.1016/j.ccell.2017.05.005

3. Chung TK, Lau TS, Cheung TH, Yim SF, Lo KW, Siu NS et al (2012) Dysregulation of microRNA-204 mediates migration and invasion of endometrial cancer by regulating FOXC1. Int J Cancer 130:1036-1045. https://doi.org/10.1002/ijc.26060

4. Conte I, Carrella S, Avellino R, Karali M, Marco-Ferreres R, Bovolenta P et al (2010) miR-204 is required for lens and retinal development via Meis2 targeting. Proc Natl Acad Sci U S A 107:15491-15496. https://doi.org/10. 1073/pnas.0914785107

5. Di Leva G, Croce CM (2013) miRNA profiling of cancer. Curr Opin Genet Dev 23:3-11. https://doi.org/10.1016/j.gde.2013.01.004

6. Ferretti E, De Smaele E, Po A, Di Marcotullio L, Tosi E, Espinola MS et al (2009) MicroRNA profiling in human medulloblastoma. Int J Cancer 124: 568-577

7. Fiaschetti G, Abela L, Nonoguchi N, Dubuc AM, Remke M, Boro A et al (2014) Epigenetic silencing of miRNA-9 is associated with HES1 oncogenic activity and poor prognosis of medulloblastoma. Br J Cancer 110:636-647. https://doi.org/10.1038/bjc.2013.764

8. Galavotti S, Bartesaghi S, Faccenda D, Shaked-Rabi M, Sanzone S, McEvoy A et al (2013) The autophagy-associated factors DRAM1 and p62 regulate cell migration and invasion in glioblastoma stem cells. Oncogene 32:699-712. https://doi.org/10.1038/onc.2012.111

9. Garancher A, Lin CY, Morabito M, Richer W, Rocques N, Larcher M et al (2018) NRL and CRX define photoreceptor identity and reveal subgroupspecific dependencies in Medulloblastoma. Cancer Cell 33:435-449 e436. https://doi.org/10.1016/j.ccell.2018.02.006

10. Garzia L, Andolfo I, Cusanelli E, Marino N, Petrosino G, De Martino D et al (2009) MicroRNA-199b-5p impairs cancer stem cells through negative regulation of HES1 in medulloblastoma. PLoS One 4:e4998. https://doi.org/ 10.1371/journal.pone.0004998

11. Gibson P, Tong Y, Robinson G, Thompson MC, Currle DS, Eden C et al (2010) Subtypes of medulloblastoma have distinct developmental origins. Nature 468:1095-1099. https://doi.org/10.1038/nature09587

12. Glick D, Barth S, Macleod KF (2010) Autophagy: cellular and molecular mechanisms. J Pathol 221:3-12. https://doi.org/10.1002/path.2697

13. Gokhale A, Kunder R, Goel A, Sarin R, Moiyadi A, Shenoy A et al (2010) Distinctive microRNA signature of medulloblastomas associated with the WNT signaling pathway. J Cancer Res Ther 6:521-529. https://doi.org/10. 4103/0973-1482.77072

14. Guo JY, Xia B, White E (2013) Autophagy-m.ediated tumor promotion. Cell 155:1216-1219. https://doi.org/10.1016/j.cell.2013.11.019

15. Guo W, Zhang Y, Zhang Y, Shi Y, Xi J, Fan H, et al (2015) Decreased expression of miR-204 in plasma is associated with a poor prognosis in patients with non-small cell lung cancer. Int J Mol Med 36: 1720-1726 Doi https://doi.org/10.3892/ijmm.2015.2388

16. Ivanov DP, Coyle B, Walker DA, Grabowska AM (2016) In vitro models of medulloblastoma: choosing the right tool for the job. J Biotechnol 236:1025. https://doi.org/10.1016/j.jbiotec.2016.07.028

17. King AA, Seidel K, Di C, Leisenring WM, Perkins SM, Krull KR et al (2017) Longterm neurologic health and psychosocial function of adult survivors of childhood
medulloblastoma/PNET: a report from the childhood Cancer survivor study. Neuro Oncol 19:689-698. https://doi.org/10.1093/neuonc/now242

18. Klionsky DJ, Abdelmohsen K, Abe A, Abedin MJ, Abeliovich H, Acevedo Arozena A et al (2016) Guidelines for the use and interpretation of assays for monitoring autophagy (3rd edition). Autophagy 12:1-222. https://doi. org/10.1080/15548627.2015.1100356

19. Kunder R, Jalali R, Sridhar E, Moiyadi A, Goel N, Goel A et al (2013) Real-time $P C R$ assay based on the differential expression of microRNAs and proteincoding genes for molecular classification of formalin-fixed paraffin embedded medulloblastomas. Neuro-Oncology 15:1644-1651. https://doi. org/10.1093/neuonc/not123

20. Lee SJ, Krauthauser C, Maduskuie V, Fawcett PT, Olson JM, Rajasekaran SA (2011) Curcumin-induced HDAC inhibition and attenuation of medulloblastoma growth in vitro and in vivo. BMC Cancer 11:144. https:// doi.org/10.1186/1471-2407-11-144

21. Lee $Y H$, Andersen JB, Song HT, Judge AD, Seo D, Ishikawa T et al (2010) Definition of ubiquitination modulator COP1 as a novel therapeutic target in human hepatocellular carcinoma. Cancer Res 70:8264-8269. https://doi. org/10.1158/0008-5472.CAN-10-0749

22. Li G, Luna C, Qiu J, Epstein DL, Gonzalez P (2011) Role of miR-204 in the regulation of apoptosis, endoplasmic reticulum stress response, and inflammation in human trabecular meshwork cells. Invest Ophthalmol Vis Sci 52:2999-3007. https://doi.org/10.1167/iovs.10-6708

23. Li W, Jin X, Zhang Q, Zhang G, Deng X, Ma L (2014) Decreased expression of miR-204 is associated with poor prognosis in patients with breast cancer. Int J Clin Exp Pathol 7:3287-3292

24. Lock R, Kenific CM, Leidal AM, Salas E, Debnath J (2014) Autophagy-dependent production of secreted factors facilitates oncogenic RAS-driven invasion. Cancer Discov 4:466-479. https://doi.org/10.1158/2159-8290.CD-13-0841

25. Louis DN, Ohgaki H, Wiestler OD, Cavenee WK, Burger PC, Jouvet A et al (2007) The 2007 WHO classification of tumours of the central nervous system. Acta Neuropathol 114:97-109. https://doi.org/10.1007/s00401-007-0243-4

26. Luzio JP, Pryor PR, Bright NA (2007) Lysosomes: fusion and function. Nat Rev Mol Cell Biol 8:622-632. https://doi.org/10.1038/nrm2217

27. Mao J, Zhang M, Zhong M, Zhang Y, Lv K (2014) MicroRNA-204, a direct negative regulator of ezrin gene expression, inhibits glioma cell migration and invasion. Mol Cell Biochem 396:117-128. https://doi.org/10.1007/ s1 1010-014-2148-6

28. Mikhaylova O, Stratton Y, Hall D, Kellner E, Ehmer B, Drew AF et al (2012) VHL-regulated MiR-204 suppresses tumor growth through inhibition of LC3B-mediated autophagy in renal clear cell carcinoma. Cancer Cell 21:532546. https://doi.org/10.1016/j.ccr.2012.02.019

29. Milde T, Lodrini M, Savelyeva L, Korshunov A, Kool M, Brueckner LM et al (2012) HD-MB03 is a novel group 3 medulloblastoma model demonstrating sensitivity to histone deacetylase inhibitor treatment. J Neuro-Oncol 110: 335-348. https://doi.org/10.1007/s11060-012-0978-1

30. Miyoshi H, Blomer U, Takahashi M, Gage FH, Verma IM (1998) Development of a self-inactivating lentivirus vector. J Virol 72:8150-8157

31. Mohammed CP, Rhee H, Phee BK, Kim K, Kim HJ, Lee H, et al (2016) miR-204 downregulates EphB2 in aging mouse hippocampal neurons. Aging Cell 15: 380-388 Doi https://doi.org/10.1111/acel.12444

32. Mosmann T (1983) Rapid colorimetric assay for cellular growth and survival: application to proliferation and cytotoxic assays. J Immunol Methods 65:55-63

33. Murphy BL, Obad S, Bihannic L, Ayrault O, Zindy F, Kauppinen S et al (2013) Silencing of the miR-17 92 cluster family inhibits medulloblastoma progression. Cancer Res 73:7068-7078. https://doi.org/10.1158/0008-5472. CAN-13-0927

34. Northcott PA, Buchhalter I, Morrissy AS, Hovestadt V, Weischenfeldt J, Ehrenberger T et al (2017) The whole-genome landscape of medulloblastoma subtypes. Nature 547:311-317. https://doi.org/10.1038/ nature22973

35. Northcott PA, Fernandez LA, Hagan JP, Ellison DW, Grajkowska W, Gillespie $Y$ et al (2009) The miR-17/92 polycistron is up-regulated in sonic hedgehogdriven medulloblastomas and induced by $\mathrm{N}$-myc in sonic hedgehog-treated cerebellar neural precursors. Cancer Res 69:3249-3255. https://doi.org/10. 1158/0008-5472.CAN-08-4710

36. Northcott PA, Korshunov A, Pfister SM, Taylor MD (2012) The clinical implications of medulloblastoma subgroups. Nat Rev Neurol 8:340-351

37. Northcott PA, Korshunov A, Witt H, Hielscher T, Eberhart CG, Mack S et al (2011) Medulloblastoma comprises four distinct molecular variants. J Clin Oncol 29:1408-1414 
38. Northcott PA, Shih DJ, Peacock J, Garzia L, Morrissy AS, Zichner T et al (2012) Subgroup-specific structural variation across 1,000 medulloblastoma genomes. Nature 488:49-56

39. Northcott PA, Shih DJ, Remke M, Cho YJ, Kool M, Hawkins C et al (2012) Rapid, reliable, and reproducible molecular sub-grouping of clinical medulloblastoma samples. Acta Neuropathol 123:615-626

40. Ostrom QT, Gittleman H, Fulop J, Liu M, Blanda R, Kromer C et al (2015) CBTRUS statistical report: primary brain and central nervous system tumors diagnosed in the United States in 2008-2012. Neuro-Oncology 17(Suppl 4): iv1-iv62. https://doi.org/10.1093/neuonc/nov189

41. Panwalkar P, Moiyadi A, Goel A, Shetty P, Goel N, Sridhar E et al (2015) MiR206, a cerebellum enriched miRNA is downregulated in all Medulloblastoma subgroups and its overexpression is necessary for growth inhibition of Medulloblastoma cells. J Mol Neurosci 56:673-680. https://doi.org/10.1007/ s12031-015-0548-z

42. Paylakhi SH, Moazzeni H, Yazdani S, Rassouli P, Arefian E, Jaberi E et al (2013) FOXC1 in human trabecular meshwork cells is involved in regulatory pathway that includes miR-204, MEIS2, and ITGbeta1. Exp Eye Res 111:112121. https://doi.org/10.1016/j.exer.2013.03.009

43. Pei Y, Liu KW, Wang J, Garancher A, Tao R, Esparza LA et al (2016) HDAC and PI3K antagonists cooperate to inhibit growth of MYC-driven Medulloblastoma. Cancer Cell 29:311-323. https://doi.org/10.1016/j.ccell. 2016.02.011

44. Ramaswamy V, Remke M, Bouffet E, Bailey S, Clifford SC, Doz F et al (2016) Risk stratification of childhood medulloblastoma in the molecular era: the current consensus. Acta Neuropathol 131:821-831. https://doi.org/10.1007/ s00401-016-1569-6

45. Remke M, Hielscher T, Korshunov A, Northcott PA, Bender S, Kool M et al (2011) FSTL5 is a marker of poor prognosis in non-WNT/non-SHH medulloblastoma. J Clin Oncol 29:3852-3861. https://doi.org/10.1200/JCO. 2011.36.2798

46. Remke M, Hielscher T, Northcott PA, Witt H, Ryzhova M, Wittmann A et al (2011) Adult medulloblastoma comprises three major molecular variants. J Clin Oncol 29:2717-2723. https://doi.org/10.1200/JCO.2011.34.9373

47. Ryan J, Tivnan A, Fay J, Bryan K, Meehan M, Creevey L et al (2012) MicroRNA-204 increases sensitivity of neuroblastoma cells to cisplatin and is associated with a favourable clinical outcome. Br J Cancer 107:967-976. https://doi.org/10.1038/bjc.2012.356

48. Sausen M, Leary RJ, Jones S, Wu J, Reynolds CP, Liu X et al (2013) Integrated genomic analyses identify ARID1A and ARID1B alterations in the childhood cancer neuroblastoma. Nat Genet 45:12-17. https://doi.org/10.1038/ng.2493

49. Schwalbe EC, Lindsey JC, Nakjang S, Crosier S, Smith AJ, Hicks D et al (2017) Novel molecular subgroups for clinical classification and outcome prediction in childhood medulloblastoma: a cohort study. Lancet Oncol 18: 958-971. https://doi.org/10.1016/S1470-2045(17)30243-7

50. Shen SQ, Huang LS, Xiao XL, Zhu XF, Xiong DD, Cao XM et al (2017) miR204 regulates the biological behavior of breast cancer MCF-7 cells by directly targeting FOXA1. Oncol Rep 38:368-376. https://doi.org/10.3892/or. 2017.5644

51. Shih DJ, Northcott PA, Remke M, Korshunov A, Ramaswamy V, Kool M et al (2014) Cytogenetic prognostication within medulloblastoma subgroups. J Clin Oncol 32:886-896. https://doi.org/10.1200/JCO.2013.50.9539

52. Sun Y, Yu X, Bai Q (2015) miR-204 inhibits invasion and epithelialmesenchymal transition by targeting FOXM1 in esophageal cancer. Int J Clin Exp Pathol 8:12775-12783

53. Uphoff CC, Drexler HG (2014) Detection of mycoplasma contamination in cell cultures. Curr Protoc Mol Biol 106: 28 24:21-14. https://doi.org/10.1002/ 0471142727.mb2804s106

54. Veno MT, Veno ST, Rehberg K, van Asperen JV, Clausen BH, Holm IE et al (2017) Cortical morphogenesis during embryonic development is regulated by miR-34c and miR-204. Front Mol Neurosci 10:31. https://doi.org/10.3389/ fnmol.2017.00031

55. Volinia S, Galasso M, Costinean S, Tagliavini L, Gamberoni G, Drusco A et al (2010) Reprogramming of miRNA networks in cancer and leukemia. Genome Res 20:589-599. https://doi.org/10.1101/gr.098046.109

56. Wang X, Holgado BL, Ramaswamy V, Mack S, Zayne K, Remke M et al (2018) miR miR on the wall, who's the most malignant medulloblastoma miR of them all? Neuro-Oncology 20:313-323. https://doi.org/10.1093/neuonc/nox106

57. Yin Y, Zhang B, Wang W, Fei B, Quan C, Zhang J et al (2014) miR-204-5p inhibits proliferation and invasion and enhances chemotherapeutic sensitivity of colorectal cancer cells by downregulating RAB22A. Clin Cancer Res 20:6187-6199. https://doi.org/10.1158/1078-0432.CCR-14-1030

58. Ying Z, Li Y, Wu J, Zhu X, Yang Y, Tian H et al (2013) Loss of miR-204 expression enhances glioma migration and stem cell-like phenotype. Cancer Res 73:990-999. https://doi.org/10.1158/0008-5472.CAN-12-2895

59. Yogi KSE, Goel N, Jalali R, Goel A, Moiyadi A, Thorat R, Panwalkar P, Khire A, Dasgupta A, Shetty P, Shirsat NV (2015) MiR-148a, a microRNA upregulated in the WNT subgroup tumors, inhibits invasion and tumorigenic potential of medulloblastoma cells by targeting Neuropilin 1. Oncoscience 2:334-348

\section{Ready to submit your research? Choose BMC and benefit from:}

- fast, convenient online submission

- thorough peer review by experienced researchers in your field

- rapid publication on acceptance

- support for research data, including large and complex data types

- gold Open Access which fosters wider collaboration and increased citations

- maximum visibility for your research: over $100 \mathrm{M}$ website views per year

At BMC, research is always in progress.

Learn more biomedcentral.com/submissions 